Canad. Math. Bull. Vol. 51 (4), 2008 pp. 604-617

\title{
The Invariant Subspace Problem for Non-Archimedean Banach Spaces
}

\author{
Wiesław Śliwa
}

Abstract. It is proved that every infinite-dimensional non-archimedean Banach space of countable type admits a linear continuous operator without a non-trivial closed invariant subspace. This solves a problem stated by A. C. M. van Rooij and W. H. Schikhof in 1992.

\section{Introduction}

Let $\mathbb{K}$ be a field with a non-trivial complete non-archimedean valuation $|\cdot|: \mathbb{K} \rightarrow$ $[0, \infty)$. Every infinite-dimensional (id) Banach space $E$ of countable type over $\mathbb{K}$ is isomorphic to the Banach space $c_{0}(\mathbb{K})$ of all sequences in $\mathbb{K}$ converging to 0 (with the sup-norm), [8, Theorem 3.16(ii)]. Any closed subspace $F$ of $E$ is $(1+\epsilon)$-complemented in $E$ for every $\varepsilon>0$, i.e., for every $\varepsilon>0$ there exists a linear continuous projection $P_{\varepsilon}$ from $E$ onto $F$ with $\left\|P_{\varepsilon}\right\| \leq 1+\varepsilon$ [8, Theorem 3.16(v)]. If the valuation of $\mathbb{K}$ is discrete, then any closed subspace of $c_{0}(\mathbb{K})$ is 1-complemented [8, Corollaries 2.4, 4.7].

Note that for complex Banach spaces, any closed subspace of a complex Banach space $E$ is complemented in $E$ if and only if $E$ is isomorphic to a complex Hilbert space [3].

Let $T$ be a linear operator on a linear space $E$. A linear subspace $M$ of $E$ is a non-trivial invariant subspace of $T$ if $\{0\} \neq M \neq E$ and $T(M) \subset M$. One of the most famous problems of the operator theory is the invariant subspace problem for complex Hilbert spaces. It asks whether every linear continuous operator on an infinite-dimensional separable complex Hilbert space has a non-trivial closed invariant subspace. This problem is still open. There exists a vast literature dedicated to the invariant subspace problem for various important classes of complex Banach spaces and linear continuous operators.

P. Enflo [2] and C. J. Read [5-7] negatively solved the invariant subspace problem for complex Banach spaces. Read obtained a linear continuous operator on the complex Banach space $l_{1}$ without a non-trivial closed invariant subspace. This $l_{1}$-example was simplified by A. M. Davie and can be found in Beauzamy's book [1, Ch. XIV].

Developing this example, we shall construct a linear continuous operator $T$ on some infinite-dimensional Banach space $E$ of countable type over $\mathbb{K}$ with no nontrivial closed invariant subspace. Clearly, $E$ is isomorphic to the Banach space $c_{0}(\mathbb{K})$. In particular, we solve the problem stated by A. C. M. van Rooij and W. H. Schikhof

\footnotetext{
Received by the editors March 14, 2006.

AMS subject classification: Primary: 47S10; secondary: 46S10, 47A15.

Keywords: invariant subspaces, non-archimedean Banach spaces.

(c) Canadian Mathematical Society 2008.
} 
in [9]: if $\mathbb{K}$ is algebraically closed and spherically complete, does every linear continuous operator $T$ on a Banach space $E$ over $\mathbb{K}$ have a non-trivial closed subspace?

Note that the Banach space $E$ of countable type is reflexive if $\mathbb{K}$ is not spherically complete [8, Corollary 4.18]. Meanwhile, for reflexive complex Banach spaces, it is not known if there exists a linear continuous operator without a non-trivial closed invariant subspace on an infinite-dimensional reflexive complex Banach space.

If $\mathbb{K}$ is algebraically closed, then it is easy to see, as in the complex case, that every linear operator $T$ on a finite-dimensional Banach space with $\operatorname{dim} E>1$ has a nontrivial invariant subspace.

If $\mathbb{K}$ is not algebraically closed (for example if $\mathbb{K}=\left(\mathbb{O}_{p}\right.$ ), we can take an element $a \in(\tilde{\mathbb{K}} \backslash \mathbb{K})$, where $\mathbb{K}$ is the algebraic closure of $\mathbb{K}$. Clearly, $E=\mathbb{K}(a)$ is a finitedimensional linear space over $\mathbb{K}$ with $\operatorname{dim} E>1$ and the linear operator $T: E \rightarrow$ $E, T y=a y$, has no non-trivial invariant subspace [9].

If $\mathbb{K}$ is not spherically complete (for example if $\mathbb{K}=\mathbb{C}_{p}$ ), then (see $[8$, Theorem 4.49]) there exists a spherically complete valued field $\hat{\mathbb{K}}$ that is an immediate extension of $\mathbb{K}$, i.e., $\mathbb{K}$ is a subfield of $\hat{K}$ and no non-zero element of $\mathbb{K}$ is orthogonal to $\mathbb{K}$. We can take an element $b \in(\hat{\mathbb{K}} \backslash \mathbb{K})$. Clearly, the closed linear span of the set $B=\left\{b^{k}: k \geq 0\right\}$ in $\hat{\mathbb{K}}$ is a Banach algebra $E$ of countable type over $\mathbb{K}$ with $\operatorname{dim} E>1$ (we consider $\hat{\mathbb{K}}$ as a Banach algebra over $\mathbb{K}$ ). In fact $E$ is a subfield of $\hat{K}$ : any non-zero element $y \in E$ is not orthogonal to $\mathbb{K}$, so there exists $z \in \mathbb{K}$ with $\|y-z\|<\max \{\|y\|,\|z\|\}$. Then $\|y\|=\|z\|$ and $\left\|1-y z^{-1}\right\|<1$. Thus $z y^{-1}=\sum_{n=0}^{\infty}\left(1-y z^{-1}\right)^{n} \in E$, so $y^{-1} \in E$. The linear continuous operator $T: E \rightarrow E, T y=b y$ has no non-trivial closed invariant subspace. Indeed, let $M$ be a closed invariant subspace of $T$. Then $V=\{y \in E: y M \subset M\}$ is a closed linear subspace of $E$ and $B \subset E$; so $V=E$. Thus $M$ is an ideal of $E$. Hence $M=\{0\}$ or $M=E[9]$.

\section{Preliminaries}

Let $K$ be a field. A function $|\cdot|: K \rightarrow[0, \infty)$ is a valuation if:

(i) $\forall \alpha \in K:|\alpha|=0 \Leftrightarrow \alpha=0$;

(ii) $\forall \alpha, \beta \in K:|\alpha \beta|=|\alpha||\beta|$;

(iii) $\forall \alpha, \beta \in K:|\alpha+\beta| \leq|\alpha|+|\beta|$.

A valuation $|\cdot|$ on $K$ is

- non-trivial if $|\alpha|>1$ for some $\alpha \in K$;

- complete if metric $d: K \times K \rightarrow[0, \infty), d(x, y)=|x-y|$ is complete on $K$;

- archimedean if the sequence $|1|,|1+1|,|1+1+1|, \ldots$ is unbounded in $[0, \infty)$;

- non-archimedean if it is not archimedean;

- discrete if the set $|K|=\{|\alpha|: \alpha \in(K \backslash\{0\})\}$ is discrete in $(0, \infty)$.

Any field with a complete archimedean valuation is topologically isomorphic to $(\mathbb{R},|\cdot|)$ or $(\mathbb{C},|\cdot|)[8$, p. 4]. A valuation $|\cdot|$ on $K$ is non-archimedean if and only if $\forall \alpha, \beta \in K:|\alpha+\beta| \leq \max \{|\alpha|,|\beta|\}[8$, Theorem 1.1].

A field $K$ with a non-trivial complete non-archimedean valuation $|\cdot|$ is called non-archimedean. The field $\mathbb{O}_{p}$ of $p$-adic numbers is non-archimedean for any 
prime number $p$.

Let $\mathbb{K}$ be a non-archimedean field. We say that $\mathbb{K}$ is spherically complete if any decreasing sequence of closed balls in $\mathbb{K}$ has non-empty intersection. An element $y \in \mathbb{K}$ is orthogonal to a subfield $L$ if $|y-z|=\max \{|y|,|z|\}$ for any $z \in L$.

By a norm on a linear space $E$ over $\mathbb{K}$ we mean a function $\|\cdot\|: E \rightarrow[0, \infty)$ such that

(i) $\forall y \in E:\|y\|=0 \Leftrightarrow y=0$;

(ii) $\forall \alpha \in \mathbb{K}, x \in E:\|\alpha x\|=\mid \alpha\|x\|$;

(iii) $\forall x, y \in E:\|x+y\| \leq \max \{\|x\|,\|y\|\}$.

If $\|\cdot\|$ is a norm on a linear space $E$ over $\mathbb{K}$ and $x, y \in E$ with $\|x\| \neq\|y\|$, then $\|x+y\|=\max \{\|x\|,\|y\|\}$.

For fundamentals of non-archimedean normed spaces we refer to $[4,8,10]$.

A normed space $E=(E,\|\cdot\|)$ is of countable type if $E$ contains a linearly dense countable set. We say that the normed spaces $E$ and $F$ over $\mathbb{K}$ are isomorphic if there is a linear bijective map $T: E \rightarrow F$ such that the maps $T$ and $T^{-1}$ are continuous.

A Banach space is a complete normed space. A series $\sum_{n=1}^{\infty} x_{n}$ in a Banach space is convergent if and only if $\lim x_{n}=0$. Every $n$-dimensional normed space $E$ over $\mathbb{K}$ is isomorphic to the Banach space $\mathbb{K}^{n}$ (with the sup-norm) and any linear operator on $E$ is continuous.

The closed graph theorem, the open mapping theorem and the Banach-Steinhaus theorem [4, Theorems 2.49, 2.73, 3.37] hold for continuous linear operators between such Banach spaces.

Let $E=(E,\|\cdot\|)$ be a normed space. A sequence $\left(y_{n}\right)_{n=0}^{\infty}$ in $E$ is a Schauder basis in $E$ if each $y \in E$ can be written uniquely as $y=\sum_{n=0}^{\infty} \alpha_{n} y_{n}$ with $\left(\alpha_{n}\right)_{n=0}^{\infty} \subset \mathbb{K}$ and the coefficient functionals $g_{n}: E \rightarrow \mathbb{K}, y \rightarrow \alpha_{n}\left(n \in \mathbb{N}_{0}\right)$ are continuous; $\mathbb{N}_{0}=\mathbb{N} \cup\{0\}$.

A linearly dense sequence $\left(y_{n}\right)_{n=0}^{\infty}$ in $E$ is an orthonormal basis in $E$ if

$$
\left\|\sum_{i=0}^{n} c_{i} y_{i}\right\|=\max _{0 \leq i \leq n}\left|c_{i}\right|
$$

for all $n \in \mathbb{N}$ and $c_{1}, \ldots, c_{n} \in \mathbb{K}$.

Any orthonormal basis in $E$ is a Schauder basis in $E$ and every Banach space with an orthonormal basis $\left(y_{n}\right)_{n=0}^{\infty}$ is isometrically isomorphic to $c_{0}(\mathbb{K})[8$, p. 171].

We denote by $\operatorname{lin} A$ the linear span of a subset $A$ of a linear space $E$.

\section{Results}

Put $d_{0}=2$. Let $\alpha \in \mathbb{K}$ with $|\alpha| \geq 8$ and $\left(d_{n}\right) \subset \mathbb{N}$ with $d_{n} \geq|\alpha|^{2 d_{n-1}}$ for $n \in \mathbb{N}$. It is easy to see that $d_{n-1} \geq 2 n$ and $d_{n} \geq|\alpha|^{2 n} d_{n-1} \geq 4 n^{2}|\alpha| d_{n-1}$ for $n \in \mathbb{N}$. Hence we have $d_{n} \geq 2^{3 n+1}, n \in \mathbb{N}$; so $d_{n} \geq|\alpha|^{2 d_{n-1}} \geq|\alpha|^{2 n+2}, n \in \mathbb{N}$. Since the function $f(x)=x^{8 / \sqrt{x}}$ is decreasing in the interval $\left(e^{2}, \infty\right)$, we get $d_{n}^{8 / \sqrt{d_{n}}} \leq|\alpha|^{16(n+1) /|\alpha|^{n+1}}<$ $|\alpha|^{1 /(n+1)}$ for $n \in \mathbb{N}$. Thus $d_{n}^{2 n+2}<|\alpha|^{\sqrt{d_{n}} / 4}<d_{n+1}$ for $n \in \mathbb{N}$.

Put $v_{0}=0, a_{n}=d_{2 n-1}, b_{n}=d_{2 n}$ and $v_{n}=(n-1)\left(a_{n}+b_{n}\right)$ for $n \in \mathbb{N}$. Then $4 n<|\alpha|^{4 n} \leq a_{n}, a_{n}^{4 n}<|\alpha|^{\sqrt{a_{n}} / 4}<b_{n}$, and $b_{n}^{4 n}<|\alpha|^{\sqrt{b_{n}} / 4}<a_{n+1}$ for every 
$n \in \mathbb{N}$. Hence we get $8 n a_{n}<b_{n}, 8 n b_{n}<a_{n+1}, 4\left(v_{n-1}+1\right)<a_{n},|\alpha| a_{n}^{2}<|\alpha|^{\sqrt{a_{n}} / 4}$, $|\alpha| b_{n}^{2}<|\alpha|^{\sqrt{b_{n}} / 4}$, and $n^{2} a_{n}^{2}<b_{n}$ for every $n \in \mathbb{N}$.

For $a, b \in \mathbb{Z}$ we denote the set $\{k \in \mathbb{Z}: a<k \leq b\}$ by $(a, b]$; similarly we define $[a, b),[a, b]$ and $(a, b)$. For nonempty sets $A, B \subset \mathbb{N}$ we write $A<B$ if $1+\max A=$ $\min B$.

For $n, r \in \mathbb{N}$ with $n>r$ we put:

$$
\begin{gathered}
J_{n, r}=\left((r-1) a_{n}+v_{n-r}, r a_{n}\right), \quad I_{n, r}=\left[r a_{n}, r a_{n}+v_{n-r-1}\right], \\
L_{n, r}=\left((n-1) a_{n}+(r-1) b_{n}, r\left(a_{n}+b_{n}\right)\right), \quad K_{n, r}=\left[r\left(a_{n}+b_{n}\right),(n-1) a_{n}+r b_{n}\right] .
\end{gathered}
$$

These sets are non-empty and $J_{n, r}<I_{n, r}<J_{n, r+1}, L_{n, r}<K_{n, r}<L_{n, r+1}$ for $n, r \in \mathbb{N}$ with $n>r+1$ and $J_{n, n-1}<I_{n, n-1}, L_{n, n-1}<K_{n, n-1}$ for $n \geq 2$. Moreover $X_{n}:=$ $\bigcup_{r=1}^{n-1}\left(J_{n, r} \cup I_{n, r}\right)=\left(v_{n-1},(n-1) a_{n}\right], Y_{n}:=\bigcup_{r=1}^{n-1}\left(L_{n, r} \cup K_{n, r}\right)=\left((n-1) a_{n}, v_{n}\right]$ for $n \in \mathbb{N}$ with $n \geq 2$; so $X_{n}<Y_{n}<X_{n+1}$ for $n \geq 2$ and $\bigcup_{n=2}^{\infty}\left(X_{n} \cup Y_{n}\right)=\mathbb{N}$.

Let $\left(\alpha_{n}\right),\left(\beta_{n}\right) \subset \mathbb{K}$ with $\left|\alpha_{n}\right| \in\left(a_{n}|\alpha|^{-1}, a_{n}\right]$ and $\left|\beta_{n}\right| \in\left(b_{n}|\alpha|^{-1}, b_{n}\right]$ for $n \in \mathbb{N}$. Clearly $1<\left|\alpha_{n}\right|<\left|\beta_{n}\right|<\left|\alpha_{n+1}\right|$ for $n \in \mathbb{N}$.

Let $\langle a\rangle=k$ if $k \leq a<k+1$ and $k \in \mathbb{Z}$. It is easy to see that $\langle a\rangle-\langle b\rangle \leq\langle a-b\rangle+1$ for $a, b \in \mathbb{R}$.

Let $F=\mathbb{K}[x]$ and $F_{n}=\{f \in F: \operatorname{deg}(f) \leq n\}$ for $n \in \mathbb{N}_{0}$. Then $F$ is a linear algebra over $\mathbb{K}$ and $F_{n}$ is a linear subspace of $F$ for every $n \in \mathbb{N}_{0}$, where $\mathbb{N}_{0}=\mathbb{N} \cup\{0\}$. Put

$$
f_{i}= \begin{cases}x^{i} & \text { if } i=0, \\ \alpha^{\left\langle\left[(2 r-1) a_{n}-2 i\right] / \sqrt{4 a_{n}}\right\rangle} x^{i} & \text { if } i \in J_{n, r} \text { and } n, r \in \mathbb{N} \text { with } n>r, \\ \alpha_{n-r}\left(x^{i}-x^{i-a_{n}}\right) & \text { if } i \in I_{n, r} \text { and } n, r \in \mathbb{N} \text { with } n>r, \\ \alpha^{\left\langle\left[(2 r-1) b_{n}-2 i\right] / \sqrt{\left.4 b_{n}\right\rangle} x^{i}\right.} & \text { if } i \in L_{n, r} \text { and } n, r \in \mathbb{N} \text { with } n>r, \\ x^{i}-\beta_{n} x^{i-b_{n}} & \text { if } i \in K_{n, r} \text { and } n, r \in \mathbb{N} \text { with } n>r .\end{cases}
$$

Obviously, $\operatorname{lin}\left\{f_{i}: 0 \leq i \leq n\right\}=F_{n}$ for $n \in \mathbb{N}_{0}$. Thus $\left(f_{i}\right)_{i=0}^{\infty}$ is a linear base in $F$. For $f \in F$ of the form $f=\sum_{i=0}^{m} c_{i} f_{i}$, we put $\|f\|=\max _{0 \leq i \leq m}\left|c_{i}\right| ;\|\cdot\|$ is a norm on $F$. Clearly, $\left(f_{i}\right)_{i=0}^{\infty}$ is an orthonormal basis in $(F,\|\cdot\|)$.

Put $A_{m}=|\alpha|^{\sqrt{a_{m}}}$ and $B_{m}=|\alpha|^{\sqrt{a_{m}}+\sqrt{b_{m}}}$ for $m \in \mathbb{N}$. We have the following.

Lemma 1 (i) Let $m \in \mathbb{N}$ with $m \geq 2$. Then $\max _{0 \leq i \leq(m-1) a_{m}}\left\|x^{i}\right\| \leq A_{m}$ and $\max _{0 \leq i \leq v_{m}}\left\|x^{i}\right\| \leq B_{m}$.

(ii) Let $n, r \in \mathbb{N}$ with $n>r$. Then $\left\|x^{i}-x^{i-r a_{n}}\right\| \leq\left|\alpha_{n-r}^{-1}\right|$ for $i \in I_{n, r}$ and $\left\|x^{i}-\beta_{n}^{r} x^{i-r b_{n}}\right\| \leq\left|\beta_{n}\right|^{r-1}$ for $i \in K_{n, r}$.

Proof For $m \in \mathbb{N}$ we have $\left[0, m a_{m+1}\right]=\left[0, v_{m}\right] \cup \bigcup_{r=1}^{m}\left(J_{m+1, r} \cup I_{m+1, r}\right)$ and $\left[0, v_{m+1}\right]=\left[0, m a_{m+1}\right] \cup \bigcup_{r=1}^{m}\left(L_{m+1, r} \cup K_{m+1, r}\right)$.

Clearly $\left\|x^{0}\right\|=1$. Let $n, r \in \mathbb{N}$ with $n>r$. It is easy to check that $\left\|x^{i}\right\|<|\alpha|^{\sqrt{a_{n}}}$ for $i \in J_{n, r}$ and $\left\|x^{i}\right\|<|\alpha|^{\sqrt{b_{n}}}$ for $i \in L_{n, r}$.

Let $i \in I_{n, r}$. For $j \in[0, r)$ we have $i-j a_{n} \in I_{n, r-j}$, so

$$
\alpha_{n-r+j}^{-1} f_{i-j a_{n}}=x^{i-j a_{n}}-x^{i-(j+1) a_{n}} .
$$


Hence $\sum_{j=0}^{r-1} \alpha_{n-r+j}^{-1} f_{i-j a_{n}}=x^{i}-x^{i-r a_{n}}$, so

$$
\left\|x^{i}-x^{i-r a_{n}}\right\| \leq \max _{0 \leq j<r}\left|\alpha_{n-r+j}^{-1}\right|=\left|\alpha_{n-r}^{-1}\right|<1 .
$$

Then $\left\|x^{i}\right\| \leq \max \left\{1,\left\|x^{i-r a_{n}}\right\|\right\}$ and $i-r a_{n} \in\left[0, v_{n-2}\right]$.

Let $i \in K_{n, r}$. For $j \in[0, r)$ we have $i-j b_{n} \in K_{n, r-j}$, so

$$
x^{i-j b_{n}}=f_{i-j b_{n}}+\beta_{n} x^{i-(j+1) b_{n}} .
$$

Hence $x^{i}=\sum_{j=0}^{r-1} \beta_{n}^{j} f_{i-j b_{n}}+\beta_{n}^{r} x^{i-r b_{n}}$, so $\left\|x^{i}-\beta_{n}^{r} x^{i-r b_{n}}\right\| \leq \max _{0 \leq j<r}\left|\beta_{n}^{j}\right|=\left|\beta_{n}^{r-1}\right|$. Then $\left\|x^{i}\right\| \leq \max \left\{\left|\beta_{n}^{r-1}\right|,\left|\beta_{n}^{r}\right| \mid x^{i-r b_{n}} \|\right\} \leq|\alpha|^{\sqrt{b_{n}}} \max \left\{1,\left\|x^{i-r b_{n}}\right\|\right\}$ and $i-r b_{n} \in$ $\left[a_{n},(n-1) a_{n}\right]$. Hence, by induction, we get $\max _{0 \leq i \leq(m-1) a_{m}}\left\|x^{i}\right\| \leq|\alpha|^{\sqrt{a_{m}}}$ and $\max _{0 \leq i \leq v_{m}}\left\|x^{i}\right\| \leq|\alpha|^{\sqrt{a_{m}}+\sqrt{b_{m}}}$ for $m \in \mathbb{N}$ with $m \geq 2$.

Denote by $E=(E,\|\cdot\|)$ the completion of the normed space $(F,\|\cdot\|)$. Then $E$ is an infinite-dimensional Banach space of countable type and $\left(f_{i}\right)_{i=0}^{\infty}$ is an orthonormal basis in $E$; so $E$ is linearly isometric to $c_{0}(\mathbb{K})$.

Lemma 2 The linear operator $T:(F,\|\cdot\|) \rightarrow(F,\|\cdot\|), T f=x f$ is continuous and $\|T\| \leq|\alpha|$.

Proof It is enough to show that $\left\|T f_{i}\right\| \leq|\alpha|$ for any $i \in \mathbb{N}_{0}$. Since $1 \in J_{2,1}$ we have $f_{1}=\alpha^{\left\langle\left[a_{2}-2\right] / \sqrt{4 a_{2}}\right\rangle} x$. Thus $T f_{0}=x=\alpha^{-\left\langle\left[a_{2}-2\right] / \sqrt{4 a_{2}}\right\rangle} f_{1}$; hence $\left\|T f_{0}\right\| \leq 1$.

Let $i \in \mathbb{N}$. For some $n, r \in \mathbb{N}$ with $n>r$ we have $i \in J_{n, r} \cup I_{n, r} \cup L_{n, r} \cup K_{n, r}$. Consider four cases (and many subcases).

Case 1: $i \in J_{n, r}$. Then $f_{i}=\alpha^{\left\langle\left[(2 r-1) a_{n}-2 i\right] / \sqrt{4 a_{n}}\right\rangle} x^{i}$.

$1.1 \quad i<r a_{n}-1$. Then $i+1 \in J_{n, r}$, so $f_{i+1}=\alpha^{\left\langle\left[(2 r-1) a_{n}-2(i+1)\right] / \sqrt{4 a_{n}}\right\rangle} x^{i+1}$. Thus $T f_{i}=$ $\alpha^{\left\langle\left[(2 r-1) a_{n}-2 i\right] / \sqrt{4 a_{n}}\right\rangle-\left\langle\left[(2 r-1) a_{n}-2(i+1)\right] / \sqrt{4 a_{n}}\right\rangle} f_{i+1}$. Hence $\left\|T f_{i}\right\| \leq|\alpha|^{\left\langle 2 / \sqrt{4 a_{n}}\right\rangle+1}=$ $|\alpha|$.

$1.2 i=r a_{n}-1$. Then $T f_{i}=\alpha^{\left\langle\left[2-a_{n}\right] / \sqrt{4 a_{n}}\right\rangle} x^{r a_{n}}$. By Lemma 1 we have $\left\|x^{r a_{n}}-x^{0}\right\| \leq$ $\left|\alpha_{n-r}^{-1}\right| \leq 1$. Thus $\left\|x^{r a_{n}}\right\| \leq \max \left\{\left\|x^{r a_{n}}-x^{0}\right\|,\left\|x^{0}\right\|\right\}=1$. Hence $\left\|T f_{i}\right\| \leq$ $|\alpha|^{-\sqrt{a_{n}} / 4} \leq a_{n}^{-1}<1$.

Case 2: $\quad i \in I_{n, r}$. Then $f_{i}=\alpha_{n-r}\left(x^{i}-x^{i-a_{n}}\right)$ and $T f_{i}=\alpha_{n-r}\left(x^{i+1}-x^{i+1-a_{n}}\right)$.

$2.1 i<r a_{n}+v_{n-r-1}$. Then $i+1 \in I_{n, r}$, so $f_{i+1}=\alpha_{n-r}\left(x^{i+1}-x^{i+1-a_{n}}\right)$. Thus $\left\|T f_{i}\right\|=\left\|f_{i+1}\right\|=1$.

$2.2 i=r a_{n}+v_{n-r-1}$. Then $4(i+1)<(4 r+1) a_{n}<b_{n}$. Put $j=i-a_{n}+1$.

If $r<n-1$, then $i+1 \in J_{n, r+1}$; so $f_{i+1}=\alpha^{\left\langle\left[(2 r+1) a_{n}-2(i+1)\right] / \sqrt{4 a_{n}}\right\rangle} x^{i+1}$. Hence $\left\|x^{i+1}\right\|=|\alpha|^{-\left\langle\left[(2 r+1) a_{n}-2(i+1)\right] / \sqrt{4 a_{n}}\right\rangle} \leq|\alpha|^{-\left\langle\sqrt{a_{n}} / 4\right\rangle}$.

If $r=n-1$, then $i+1 \in L_{n, 1}$; so $f_{i+1}=\alpha^{\left\langle\left[b_{n}-2(i+1)\right] / \sqrt{4 b_{n}}\right\rangle} x^{i+1}$. Hence $\left\|x^{i+1}\right\|=|\alpha|^{-\left\langle\left[b_{n}-2(i+1)\right] / \sqrt{4 b_{n}}\right\rangle} \leq|\alpha|^{-\left\langle\sqrt{b_{n}} / 4\right\rangle}$.

If $r=1$ and $n=2$, then $j=1 \in J_{2,1}$ and $\left\|x^{j}\right\|=|\alpha|^{-\left\langle\left[a_{2}-2\right] / \sqrt{4 a_{2}}\right\rangle}\left\|f_{j}\right\| \leq$ $|\alpha|^{-\left\langle\sqrt{a_{2}} / 4\right\rangle}$. 
If $r=1$ and $n>2$, then $j \in J_{n-1,1}$; so $f_{j}=\alpha^{\left\langle\left[a_{n-1}-2 j\right] / \sqrt{4 a_{n-1}}\right\rangle} x^{j}$. Hence $\left\|x^{j}\right\|=|\alpha|^{-\left\langle\left[a_{n-1}-2\left(v_{n-2}+1\right)\right] / \sqrt{4 a_{n-1}}\right\rangle} \leq|\alpha|^{-\left\langle\sqrt{a_{n-1}} / 4\right\rangle}$.

If $1<r<n-1$, then $j \in I_{n, r-1}$ and using Lemma 1 we get $\left\|x^{j}-x^{v_{n-r-1}+1}\right\| \leq$ $\left|\alpha_{n-r+1}^{-1}\right|$. Moreover we have $f_{v_{n-r-1}+1}=\alpha^{\left\langle\left[a_{n-r}-2\left(v_{n-r-1}+1\right)\right] / \sqrt{4 a_{n-r}}\right\rangle} x^{v_{n-r-1}+1}$, because $v_{n-r-1}+1 \in J_{n-r, 1}$. Hence

$$
\left\|x^{v_{n-r-1}+1}\right\|=|\alpha|^{-\left\langle\left[a_{n-r}-2\left(v_{n-r-1}+1\right)\right] / \sqrt{4 a_{n-r}}\right\rangle} \leq|\alpha|^{-\left\langle\sqrt{a_{n-r}} / 4\right\rangle} .
$$

Thus $\left\|x^{j}\right\| \leq \max \left\{\left\|x^{j}-x^{v_{n-r-1}+1}\right\|,\left\|x^{v_{n-r-1}+1}\right\|\right\} \leq|\alpha|^{-\left\langle\sqrt{a_{n-r}} / 4\right\rangle}$.

If $r=n-1$, then $j \in J_{n, n-1}$; so $f_{j}=\alpha^{\left\langle\left[(2 n-3) a_{n}-2 j\right] / \sqrt{4 a_{n}}\right\rangle} x^{j}$. Hence $\left\|x^{j}\right\|=$ $|\alpha|^{-\left\langle\left[a_{n}-2\right] / \sqrt{4 a_{n}}\right\rangle} \leq|\alpha|^{-\left\langle\sqrt{a_{n}} / 4\right\rangle}$.

It follows that $\left\|T f_{i}\right\| \leq\left|\alpha_{n-r}\right| \max \left\{\left\|x^{i+1}\right\|,\left\|x^{j}\right\|\right\} \leq\left|\alpha_{n-r}\right||\alpha|^{-\left\langle\sqrt{a_{n-r}} / 4\right\rangle} \leq$ $a_{n-r}^{-1}$.

Case 3: $i \in L_{n, r}$. Then $f_{i}=\alpha^{\left\langle\left[(2 r-1) b_{n}-2 i\right] / \sqrt{4 b_{n}}\right\rangle} x^{i}$.

$3.1 i<r\left(a_{n}+b_{n}\right)-1$. Then $i+1 \in L_{n, r}$, so $f_{i+1}=\alpha^{\left\langle\left[(2 r-1) b_{n}-2(i+1)\right] / \sqrt{4 b_{n}}\right\rangle} x^{i+1}$. Thus $T f_{i}=\alpha^{\left\langle\left[(2 r-1) b_{n}-2 i\right] / \sqrt{4 b_{n}}\right\rangle-\left\langle\left[(2 r-1) b_{n}-2(i+1)\right] / \sqrt{4 b_{n}}\right\rangle} f_{i+1}$. Hence $\left\|T f_{i}\right\| \leq|\alpha|$.

$3.2 i=r\left(a_{n}+b_{n}\right)-1$. Then $T f_{i}=\alpha^{\left\langle\left[-b_{n}-2 r a_{n}+2\right] / \sqrt{4 b_{n}}\right\rangle} x^{r\left(a_{n}+b_{n}\right)}$. Put $j=r\left(a_{n}+b_{n}\right)$. By Lemma 1 we have $\left\|x^{j}-\beta_{n}^{r} x^{j-r b_{n}}\right\| \leq\left|\beta_{n}\right|^{r-1}$. In Case 1 we have shown that $\left\|x^{r a_{n}}\right\| \leq 1$. Hence $\left\|x^{j}\right\| \leq \max \left\{\left\|x^{j}-\beta_{n}^{r} x^{r a_{n}}\right\|,\left|\beta_{n}^{r}\right|\left\|x^{r a_{n}}\right\|\right\} \leq b_{n}^{n-1}$. Thus $\left\|T f_{i}\right\| \leq|\alpha|^{\left\langle\left[-b_{n}-2 r a_{n}+2\right] / \sqrt{4 b_{n}}\right\rangle} b_{n}^{n-1} \leq|\alpha|^{-\sqrt{b_{n}} / 4} b_{n}^{n-1} \leq b_{n}^{-1}$.

Case 4: $i \in K_{n, r}$. Then $f_{i}=x^{i}-\beta_{n} x^{i-b_{n}}$ and $T f_{i}=x^{i+1}-\beta_{n} x^{i+1-b_{n}}$.

$4.1 i<(n-1) a_{n}+r b_{n}$. Then $i+1 \in K_{n, r}$, so $f_{i+1}=x^{i+1}-\beta_{n} x^{i+1-b_{n}}=T f_{i}$. Hence $\left\|T f_{i}\right\|=1$.

$4.2 i=(n-1) a_{n}+r b_{n}$. Put $j=i+1-b_{n}$. Then $j \in L_{n, r}$, so

$$
f_{j}=\alpha^{\left\langle\left[(2 r-1) b_{n}-2 j\right] / \sqrt{4 b_{n}}\right\rangle} x^{j}
$$

Hence $\left\|x^{j}\right\|=|\alpha|^{-\left\langle\left[(2 r+1) b_{n}-2(i+1)\right] / \sqrt{4 b_{n}}\right\rangle} \leq|\alpha|^{-\left\langle\sqrt{b_{n}} / 4\right\rangle}$.

If $r<n-1$, then $i+1 \in L_{n, r+1}$; so $f_{i+1}=\alpha^{\left\langle\left[(2 r+1) b_{n}-2(i+1)\right] / \sqrt{4 b_{n}}\right\rangle} x^{i+1}$. Hence $\left\|x^{i+1}\right\|=\left\|x^{j}\right\| \leq|\alpha|^{-\left\langle\sqrt{b_{n}} / 4\right\rangle}$.

If $r=n-1$, then $i+1 \in J_{n+1,1}$; so $f_{i+1}=\alpha^{\left\langle\left[a_{n+1}-2(i+1)\right] / \sqrt{4 a_{n+1}}\right\rangle} x^{i+1}$. Hence $\left\|x^{i+1}\right\|=|\alpha|^{-\left\langle\left[a_{n+1}-2(i+1)\right] / \sqrt{4 a_{n+1}}\right\rangle} \leq|\alpha|^{-\left\langle\sqrt{a_{n+1}} / 4\right\rangle} \leq|\alpha|^{-\left\langle\sqrt{b_{n}} / 4\right\rangle}$. Thus we have $\left\|T f_{i}\right\| \leq \max \left\{\left\|x^{i+1}\right\|,\left|\beta_{n}\right|\left\|x^{j}\right\|\right\} \leq\left|\beta_{n}\right||\alpha|^{-\left\langle\sqrt{b_{n}} / 4\right\rangle} \leq|\alpha|$.

From now on, by $T$ we will denote the linear continuous operator on $E$ such that $T f=x f$ for all $f \in F$; clearly $\|T\| \leq|\alpha|$.

By the proof of Lemma 2 we get the following.

Remark 3. If $n, r \in \mathbb{N}$ with $n>r$, then $\left\|T f_{r a_{n}-1}\right\| \leq a_{n}^{-1},\left\|T f_{r a_{n}+v_{n-r-1}}\right\| \leq a_{n-r}^{-1}$, and $\left\|T f_{r\left(a_{n}+b_{n}\right)-1}\right\| \leq b_{n}^{-1}$.

Let $m \in \mathbb{N}$ with $m>2$. Put $S_{m}=\bigcup_{n=m+1}^{\infty} I_{n, n-m}$. Let $Q_{m}: F \rightarrow F_{(m-1) a_{m}}$ be a 
linear operator such that

$$
Q_{m} f_{i}= \begin{cases}f_{i} & \text { if } i \in\left[0,(m-1) a_{m}\right] \\ -\alpha_{m} x^{i-(n-m) a_{n}} & \text { if } i \in I_{n . n-m} \text { and } n>m, \\ 0 & \text { if } i \in\left(\mathbb{N} \backslash S_{m}\right) \text { with } i>(m-1) a_{m}\end{cases}
$$

Clearly, $\left\|Q_{m} f_{i}\right\|=1$ for $0 \leq i \leq(m-1) a_{m}$, and $\left\|Q_{m} f_{i}\right\|=\left|\alpha_{m}\left\|\mid x^{i-(n-m) a_{n}}\right\| \leq\right.$ $a_{m} B_{m-1}<\sqrt{A_{m}}$ for $i \in I_{n, n-m}, n>m$. Thus $\sup _{i \in \mathbb{N}_{0}}\left\|Q_{m} f_{i}\right\|<\sqrt{A_{m}}$; so the linear operator $Q_{m}:(F,\|\cdot\|) \rightarrow(F,\|\cdot\|)$ is continuous and $\left\|Q_{m}\right\|<\sqrt{A_{m}}$. From now on, by $Q_{m}$ we will denote its continuous extension on $(E,\|\cdot\|)$.

We have the following lemma.

Lemma 4 Let $m \in \mathbb{N}$ with $m>2$ and let $s \in K_{m, 1}$. Then $\left\|T^{s}-T^{s} Q_{m}\right\| \leq|\alpha|$.

Proof It is enough to show that $\left\|T^{s} f_{i}-T^{s} Q_{m} f_{i}\right\| \leq|\alpha|$ for every $i \in \mathbb{N}_{0}$. If $i \in$ $\left[0,(m-1) a_{m}\right]$, then $T^{s} f_{i}-T^{s} Q_{m} f_{i}=0$. Let $i>(m-1) a_{m}$. For some $n, r \in \mathbb{N}$ with $n>r$ we have $i \in J_{n, r} \cup I_{n, r} \cup L_{n, r} \cup K_{n, r}$. If $i \in J_{n, r} \cup L_{n, r} \cup K_{n, r}$, then $i \notin S_{m}$; so $Q_{m} f_{i}=0$ and $T^{s} f_{i}-T^{s} Q_{m} f_{i}=T^{s} f_{i}$. Consider four cases.

Case 1: $i \in J_{n, r}$. Then $T^{s} f_{i}=\alpha^{\left\langle\left[(2 r-1) a_{n}-2 i\right] / \sqrt{4 a_{n}}\right\rangle} x^{i+s}$. We have $m<n$, since $(m-1) a_{m}<i<(n-1) a_{n}$. Thus $s<2 b_{m}<\sqrt{a_{n}}$.

$1.1 i<r a_{n}-s$. Then $i+s \in J_{n, r}$, so $f_{i+s}=\alpha^{\left\langle\left[(2 r-1) a_{n}-2(i+s)\right] / \sqrt{4 a_{n}}\right\rangle} x^{i+s}$. Hence $T^{s} f_{i}=\alpha^{\left\langle\left[(2 r-1) a_{n}-2 i\right] / \sqrt{4 a_{n}}\right\rangle-\left\langle\left[(2 r-1) a_{n}-2(i+s)\right] / \sqrt{4 a_{n}}\right\rangle} f_{i+s}$. Thus $\left\|T^{s} f_{i}\right\| \leq|\alpha|$.

$1.2 i \geq r a_{n}-s$. Then $T^{i+s-r a_{n}+1} f_{r a_{n}-1}=\alpha^{\left\langle\left[(2 r-1) a_{n}-2\left(r a_{n}-1\right)\right] / \sqrt{4 a_{n}}\right\rangle} x^{i+s}$, since $r a_{n}-1 \in J_{n, r}$. Thus

$$
\begin{aligned}
\left\|T^{s} f_{i}\right\| & =|\alpha|^{\left\langle\left[(2 r-1) a_{n}-2 i\right] / \sqrt{4 a_{n}}\right\rangle-\left\langle\left[-a_{n}+2\right] / \sqrt{4 a_{n}}\right\rangle}\left\|T^{i+s-r a_{n}+1} f_{r a_{n}-1}\right\| \\
& \leq|\alpha|\|T\|^{i+s-r a_{n}}\left\|T f_{r a_{n}-1}\right\| .
\end{aligned}
$$

By Remark 3 we have $\left\|T f_{r a_{n}-1}\right\| \leq a_{n}^{-1}$. It follows that

$$
\left\|T^{s} f_{i}\right\| \leq|\alpha|^{1+i+s-r a_{n}} a_{n}^{-1} \leq|\alpha|^{s} a_{n}^{-1} \leq|\alpha|^{2 b_{m}} a_{m+1}^{-1} \leq 1
$$

Case 2: $\quad i \in I_{n, r}$. Then $f_{i}=\alpha_{n-r}\left(x^{i}-x^{i-a_{n}}\right)$, so $T^{s} f_{i}=\alpha_{n-r}\left(x^{i+s}-x^{i+s-a_{n}}\right)$. We have $n>m$, since $(m-1) a_{m}<i \leq r a_{n}+v_{n-r-1} \leq(n-1) a_{n}$. Thus $4(i+s)<(4 r+1) a_{n}$.

$2.1 r=n-m$. Then $i=r a_{n}+l$ for some $l \in\left[0, v_{m-1}\right]$ and $T^{s} Q_{m} f_{i}=-\alpha_{m} x^{l+s}$. We have $i+s \in J_{n, r+1}$, so $f_{i+s}=\alpha^{\left\langle\left[(2 r+1) a_{n}-2(i+s)\right] / \sqrt{\left.4 a_{n}\right\rangle}\right.} x^{i+s}$. Hence $\left\|x^{i+s}\right\|=$ $|\alpha|^{-\left\langle\left[(2 r+1) a_{n}-2(i+s)\right] / \sqrt{4 a_{n}}\right\rangle} \leq|\alpha|^{-\left\langle\sqrt{a_{n}} / 4\right\rangle} \leq\left|\alpha_{n}^{-1}\right|$. Using Lemma 1 we get

$$
\left\|x^{(r-1) a_{n}+l+s}-x^{l+s}\right\| \leq\left|\alpha_{n-r+1}^{-1}\right|=\left|\alpha_{m+1}^{-1}\right| .
$$

Thus $\left\|T^{s} f_{i}-T^{s} Q_{m} f_{i}\right\|=\left|\alpha_{m}\left\|\mid x^{i+s}-\left(x^{i-a_{n}+s}-x^{l+s}\right)\right\|<1\right.$.

$2.2 r \neq n-m$. Then $i \notin S_{m}$, so $Q_{m} f_{i}=0$ and $T^{s} f_{i}-T^{s} Q_{m} f_{i}=T^{s} f_{i}$. 
2.2a $\quad r>n-m$. Then for $j=i+s$ we have $j-a_{n} \in J_{n, r}$, so $f_{j-a_{n}}=$ $\alpha^{\left\langle\left[(2 r-1) a_{n}-2\left(j-a_{n}\right)\right] / \sqrt{4 a_{n}}\right\rangle} x^{j-a_{n}}$. Thus $\left\|x^{j-a_{n}}\right\|=|\alpha|^{-\left\langle\left[(2 r+1) a_{n}-2 j\right] / \sqrt{4 a_{n}}\right\rangle} \leq$ $|\alpha|^{-\left\langle\sqrt{a_{n}} / 4\right\rangle}$.

If $r+1<n$, then $j \in J_{n, r+1}$; so $f_{j}=\alpha^{\left\langle\left[(2 r+1) a_{n}-2 j\right] / \sqrt{4 a_{n}}\right\rangle} x^{j}$. Hence $\left\|x^{j}\right\|=\left\|x^{j-a_{n}}\right\| \leq|\alpha|^{-\left\langle\sqrt{a_{n}} / 4\right\rangle}$.

If $r+1=n$, then $j \in L_{n, 1}$; so $f_{j}=\alpha^{\left\langle\left[b_{n}-2 j\right] / \sqrt{4 b_{n}}\right\rangle} x^{j}$. Hence $\left\|x^{j}\right\|=$ $|\alpha|^{-\left\langle\left[b_{n}-2 j\right] / \sqrt{4 b_{n}}\right\rangle} \leq|\alpha|^{-\left\langle\sqrt{b_{n}} / 4\right\rangle}$.

It follows that

$\left\|T^{s} f_{i}\right\| \leq\left|\alpha_{n-r}\right| \max \left\{\left\|x^{j}\right\|,\left\|x^{j-a_{n}}\right\|\right\} \leq\left|\alpha_{n} \| \alpha\right|^{-\left\langle\sqrt{a_{n}} / 4\right\rangle}<1$.

2.2b $\quad r<n-m$. Then $i=r a_{n}+l$ for some $l \in\left[0, v_{n-r-1}\right]$.

If $l+s \leq v_{n-r-1}$, then $i+s \in I_{n, r}$; so $f_{i+s}=\alpha_{n-r}\left(x^{i+s}-x^{i+s-a_{n}}\right)=T^{s} f_{i}$. Thus $\left\|T^{s} f_{i}\right\|=1$.

If $l+s>v_{n-r-1}$, then we have

$T^{s} f_{i}=\alpha_{n-r}\left(x^{i+s}-x^{i+s-a_{n}}\right)=T^{s+l-v_{n-r-1}}\left[\alpha_{n-r}\left(x^{r a_{n}+v_{n-r-1}}-x^{(r-1) a_{n}+v_{n-r-1}}\right)\right]$

$=T^{s+l-v_{n-r-1}-1} T f_{r a_{n}+v_{n-r-1}}$.

By Remark 3 we obtain $\left\|T f_{r a_{n}+v_{n-r-1}}\right\| \leq a_{n-r}^{-1}$. Thus we get $\left\|T^{s} f_{i}\right\| \leq$ $\|T\|^{s+l-v_{n-r-1}-1} a_{n-r}^{-1} \leq|\alpha|^{s} a_{n-r}^{-1} \leq|\alpha|^{2 b_{m}} a_{m+1}^{-1} \leq 1$.

Case 3: $\quad i \in L_{n, r}$. Then $T^{s} f_{i}=\alpha^{\left\langle\left[(2 r-1) b_{n}-2 i\right] / \sqrt{4 b_{n}}\right\rangle} x^{i+s}$. Put $j=i+s$. We have $n \geq m$, since $v_{m-1}<a_{m}<i<r\left(a_{n}+b_{n}\right) \leq v_{n}$. Thus $4 j<a_{n+1}$.

$3.1 n=m$ and $j>v_{n}$. Then $j \in J_{n+1,1}$; so $f_{j}=\alpha^{\left\langle\left[a_{n+1}-2 j\right] / \sqrt{4 a_{n+1}}\right\rangle} x^{j}$ and

$\left\|x^{j}\right\|=|\alpha|^{-\left\langle\left[a_{n+1}-2 j\right] / \sqrt{4 a_{n+1}}\right\rangle} \leq|\alpha|^{-\left\langle\sqrt{a_{n+1}} / 4\right\rangle}$. Thus $\left\|T^{s} f_{i}\right\| \leq|\alpha|^{\left\langle\sqrt{b_{n}} / 2\right\rangle}\left\|x^{j}\right\| \leq$ $|\alpha|^{\left\langle\sqrt{b_{n}} / 2\right\rangle-\left\langle\sqrt{a_{n+1}} / 4\right\rangle} \leq 1$.

$3.2 n=m$ and $j \leq v_{n}$. Then $j>n a_{n}+r b_{n}$ and $r<n-1$.

3.2a If $j<(r+1)\left(a_{n}+b_{n}\right)$, then $j \in L_{n, r+1}$; so $f_{j}=\alpha^{\left\langle\left[(2 r+1) b_{n}-2 j\right] / \sqrt{4 b_{n}}\right\rangle} x^{j}$. Thus $\left\|T^{s} f_{i}\right\|=|\alpha|^{\left\langle\left[(2 r-1) b_{n}-2 i\right] / \sqrt{4 b_{n}}\right\rangle-\left\langle\left[(2 r+1) b_{n}-2 j\right] / \sqrt{4 b_{n}}\right\rangle} \leq|\alpha|$, since $s-b_{n}<\sqrt{b_{n}}$.

3.2b If $(r+1)\left(a_{n}+b_{n}\right) \leq j \leq(n-1) a_{n}+(r+1) b_{n}$, then using Lemma 1 we get $\left\|x^{j}-\beta_{n}^{r+1} x^{j-(r+1) b_{n}}\right\| \leq\left|\beta_{n}^{r}\right| \leq b_{n}^{r}$ and $\left\|x^{j-(r+1) b_{n}}\right\| \leq A_{n}<b_{n}^{4}$. Thus $\left\|x^{j}\right\| \leq \max \left\{b_{n}^{r}, b_{n}^{r+5}\right\} \leq b_{n}^{4 n}<|\alpha|^{\sqrt{b_{n}} / 4}$. Moreover, we have

$$
\left\|T^{s} f_{i}\right\|=|\alpha|^{\left\langle\left[(2 r-1) b_{n}-2 i\right] / \sqrt{4 b_{n}}\right\rangle}\left\|x^{j}\right\| \leq|\alpha|^{\left\langle-\sqrt{b_{n}} / 4\right\rangle}\left\|x^{j}\right\|,
$$

since $i=j-s \geq[r-(1 / 4)] b_{n}$. It follows that $\left\|T^{s} f_{i}\right\| \leq 1$.

$3.2 \mathrm{c}$ If $j>(n-1) a_{n}+(r+1) b_{n}$, then $r<n-2$ and $j \in L_{n, r+2}$; so $f_{j}=$ $\alpha^{\left\langle\left[(2 r+3) b_{n}-2 j\right] / \sqrt{4 b_{n}}\right\rangle} x^{j}$. Thus

$$
\left\|T^{s} f_{i}\right\|=|\alpha|^{\left\langle\left[(2 r-1) b_{n}-2 i\right] / \sqrt{4 b_{n}}\right\rangle-\left\langle\left[(2 r+3) b_{n}-2 j\right] / \sqrt{4 b_{n}}\right\rangle} \leq 1 .
$$

3.3 $n>m$ and $j<r\left(a_{n}+b_{n}\right)$. Then $j \in L_{n, r}$, so $f_{j}=\alpha^{\left\langle\left[(2 r-1) b_{n}-2 j\right] / \sqrt{4 b_{n}}\right\rangle} x^{j}$. Thus $\left\|T^{s} f_{i}\right\|=|\alpha|^{\left\langle\left[(2 r-1) b_{n}-2 i\right] / \sqrt{4 b_{n}}\right\rangle-\left\langle\left[(2 r-1) b_{n}-2 j\right] / \sqrt{4 b_{n}}\right\rangle} \leq|\alpha|$, since $s<2 b_{m}<$ $\sqrt{b_{n}}$. 
$3.4 n>m$ and $j \geq r\left(a_{n}+b_{n}\right)$. Put $k=r\left(a_{n}+b_{n}\right)$. Clearly $k-1 \in L_{n, r}$, so $f_{k-1}=$ $\alpha^{\left\langle\left[(2 r-1) b_{n}-2(k-1)\right] / \sqrt{4 b_{n}}\right\rangle} x^{k-1}$. Hence $T^{j-k} T f_{k-1}=\alpha^{\left\langle\left[(2 r-1) b_{n}-2(k-1)\right] / \sqrt{4 b_{n}}\right\rangle} x^{j}$. Thus $\left\|x^{j}\right\| \leq|\alpha|^{-\left\langle\left[(2 r-1) b_{n}-2(k-1)\right] / \sqrt{4 b_{n}}\right\rangle}\|T\|^{s-1}\left\|T f_{k-1}\right\|$. Using Remark 3 we obtain $\left\|T f_{k-1}\right\| \leq b_{n}^{-1}$. Thus

$$
\begin{aligned}
\left\|T^{s} f_{i}\right\| & \leq|\alpha|^{\left\langle\left[(2 r-1) b_{n}-2 i\right] / \sqrt{4 b_{n}}\right\rangle-\left\langle\left[(2 r-1) b_{n}-2(k-1)\right] / \sqrt{4 b_{n}}\right\rangle}|\alpha|^{s-1} b_{n}^{-1} \\
& \leq|\alpha|^{s} b_{n}^{-1}<|\alpha|^{2 b_{m}} b_{m+1}^{-1}<1,
\end{aligned}
$$

since $k-i-1<s<2 b_{m}<\sqrt{b_{n}}$.

Case 4: $i \in K_{n, r}$. Then $f_{i}=x^{i}-\beta_{n} x^{i-b_{n}}$ and $T^{s} f_{i}=x^{i+s}-\beta_{n} x^{i+s-b_{n}}$. We have $n \geq m$, since $(m-1) a_{m}<i \leq(n-1) a_{n}+r b_{n} \leq v_{n}$. Put $j=i+s$.

$4.1 n=m$ and $j \leq(n-1) a_{n}+(r+1) b_{n}$. Then $j \geq(r+1)\left(a_{n}+b_{n}\right)$, so $r+1 \leq n-1$ and $j \in K_{n, r+1}$. Thus $f_{j}=x^{j}-\beta_{n} x^{j-b_{n}}=T^{s} f_{i}$, so $\left\|T^{s} f_{i}\right\|=1$.

$4.2 n=m$ and $j>(n-1) a_{n}+(r+1) b_{n}$. Then $4 j<(4 r+5) b_{n}<a_{n+1}$.

If $r<n-2$, then $j \in L_{n, r+2}$ and $f_{j}=\alpha^{\left\langle\left[(2 r+3) b_{n}-2 j\right] / \sqrt{4 b_{n}}\right\rangle} x^{j}$; so $\left\|x^{j}\right\|=$ $|\alpha|^{-\left\langle\left[(2 r+3) b_{n}-2 j\right] / \sqrt{4 b_{n}}\right\rangle} \leq 1$.

If $r \geq n-2$, then $j \in J_{n+1,1}$ and $f_{j}=\alpha^{\left\langle\left[a_{n+1}-2 j\right] / \sqrt{4 a_{n+1}}\right\rangle} x^{j}$; so $\left\|x^{j}\right\|=$ $|\alpha|^{-\left\langle\left[a_{n+1}-2 j\right] / \sqrt{4 a_{n+1}}\right\rangle} \leq 1$.

If $r<n-1$, then $j-b_{n} \in L_{n, r+1}$ and $f_{j-b_{n}}=\alpha^{\left\langle\left[(2 r+1) b_{n}-2\left(j-b_{n}\right)\right] / \sqrt{4 b_{n}}\right\rangle} x^{j-b_{n}}$; so $\left\|x^{j-b_{n}}\right\|=|\alpha|^{-\left\langle\left[(2 r+3) b_{n}-2 j\right] / \sqrt{4 b_{n}}\right\rangle} \leq|\alpha|^{-\left\langle\sqrt{b_{n}} / 4\right\rangle} \leq\left|\beta_{n}^{-1}\right|$.

If $r=n-1$, then $j-b_{n} \in J_{n+1,1}$ and $f_{j-b_{n}}=\alpha^{\left\langle\left[a_{n+1}-2\left(j-b_{n}\right)\right] / \sqrt{4 a_{n+1}}\right\rangle} x^{j-b_{n}}$; so $\left\|x^{j-b_{n}}\right\|=|\alpha|^{-\left\langle\left[a_{n+1}-2\left(j-b_{n}\right)\right] / \sqrt{4 a_{n+1}}\right\rangle} \leq|\alpha|^{-\left\langle\sqrt{a_{n+1}} / 4\right\rangle} \leq\left|\alpha_{n+1}^{-1}\right|$.

It follows that $\left\|T^{s} f_{i}\right\| \leq \max \left\{\left\|x^{j}\right\|,\left|\beta_{n}\left\|\mid x^{j-b_{n}}\right\|\right\} \leq 1\right.$.

$4.3 n>m$ and $j \leq(n-1) a_{n}+r b_{n}$. Then $j \in K_{n, r}$ and $f_{j}=x^{j}-\beta_{n} x^{j-b_{n}}=T^{s} f_{i}$; so $\left\|T^{s} f_{i}\right\|=1$.

$4.4 n>m$ and $j>(n-1) a_{n}+r b_{n}$. Then $4 j<(4 r+1) b_{n}<a_{n+1}$.

If $r<n-1$, then $j \in L_{n, r+1}$ and $f_{j}=\alpha^{\left\langle\left[(2 r+1) b_{n}-2 j\right] / \sqrt{4 b_{n}}\right\rangle} x^{j}$; so $\left\|x^{j}\right\|=$ $|\alpha|^{-\left\langle\left[(2 r+1) b_{n}-2 j\right] / \sqrt{4 b_{n}}\right\rangle} \leq 1$.

If $r=n-1$, then $j \in J_{n+1,1}$ and $f_{j}=\alpha^{\left\langle\left[a_{n+1}-2 j\right] / \sqrt{4 a_{n+1}}\right\rangle} x^{j}$; so $\left\|x^{j}\right\|=$ $|\alpha|^{-\left\langle\left[a_{n+1}-2 j\right] / \sqrt{4 a_{n+1}}\right\rangle} \leq 1$.

Moreover we have $j-b_{n} \in L_{n, r}$ and $f_{j-b_{n}}=\alpha^{\left\langle\left[(2 r-1) b_{n}-2\left(j-b_{n}\right)\right] / \sqrt{4 b_{n}}\right\rangle} x^{j-b_{n}}$; so $\left\|x^{j-b_{n}}\right\|=|\alpha|^{-\left\langle\left[(2 r+1) b_{n}-2 j\right] / \sqrt{4 b_{n}}\right\rangle} \leq|\alpha|^{-\left\langle\sqrt{b_{n}} / 4\right\rangle} \leq\left|\beta_{n}^{-1}\right|$. It follows that $\left\|T^{s} f_{i}\right\| \leq \max \left\{\left\|x^{j}\right\|,\left|\beta_{n}\left\|\mid x^{j-b_{n}}\right\|\right\} \leq 1\right.$.

For $f \in F$ of the form $f=\sum_{i=0}^{m} c_{i} x^{i}$ we put $|f|=\max _{0 \leq i \leq m}\left|c_{i}\right|$. The functional $|\cdot|: F \rightarrow[0, \infty), f \rightarrow|f|$ is a multiplicative norm on $F[8, \bar{p} .7]$.

It is easy to check that for $m \in \mathbb{N}$ with $m>2$ and $y \in F_{(m-1) a_{m}}$ we have $\|y\| \leq$ $A_{m}|y|$ and $|y| \leq \max _{0 \leq i \leq(m-1) a_{m}}\left|f_{i}\right|\|y\| \leq \sqrt{A_{m}}\|y\|$.

For $n \in \mathbb{N}_{0}$ we denote by $P_{n}$ the linear projection from $F$ onto $F_{n}$ such that $P_{n}\left(x^{i}\right)=0$ for $i>n$. We have $x\left(P_{n} v\right)=P_{n+1}(x v)$ for $n \in \mathbb{N}$ and $v \in F$.

We need two more lemmas to prove our theorem.

Lemma 5 Let $e \in E$ with $e \neq 0$ and $k \in \mathbb{N}$ with $k>2$. Then there exists $m \in \mathbb{N}$ with $m>k$ such that $\left|P_{(m-k) a_{m}}\left(Q_{m} e\right)\right| \geq a_{m}^{-1}$. 
Proof Suppose by contradiction that for every $m \in \mathbb{N}$ with $m>k$ we have

$$
\left|P_{(m-k) a_{m}}\left(Q_{m} e\right)\right|<a_{m}^{-1} .
$$

For some $\left(e_{j}\right) \in c_{0}(\mathbb{K})$ we have $e=\sum_{j=0}^{\infty} e_{j} f_{j}$. Then $\|e\|=\max _{j \in \mathbb{N}_{0}}\left|e_{j}\right|>0$. Put $c_{n}=(n-1) a_{n}$ for $n \in \mathbb{N}$. For $n \in \mathbb{N}$ we have $\sum_{j=0}^{c_{n}} e_{j} f_{j}=\sum_{j=0}^{c_{n}} y_{n, j} x^{j}$ for some $\left(y_{n, j}\right)_{j=0}^{c_{n}} \subset \mathbb{K}$. For $n \in \mathbb{N}$ with $n>2$ we obtain $Q_{n}\left(\sum_{j=c_{n}+1}^{\infty} e_{j} f_{j}\right)=\sum_{i=0}^{v_{n-1}} z_{n, i} x^{i}$, where

$$
z_{n, i}=-\alpha_{n} \sum_{m=n+1}^{\infty} e_{i+(m-n) a_{m}}
$$

So we get

$$
Q_{n} e=\sum_{j=0}^{c_{n}} y_{n, j} x^{j}+\sum_{j=0}^{v_{n-1}} z_{n, j} x^{j}
$$

From (3.1) and (3.3) we obtain for $m \in \mathbb{N}$ with $m>k$

$$
\max _{j \in\left(v_{m-1},(m-k) a_{m}\right]}\left|y_{m, j}\right|<a_{m}^{-1} .
$$

Let $m>n>k$ and $M_{m, n}=\left((m-n) a_{m}+v_{n-2},(m-n) a_{m}+v_{n-1}\right]$. Clearly, $M_{m, n} \subset$ $\left[a_{m}, c_{m}\right]=\bigcup_{s=1}^{m-1} I_{m, s} \cup \bigcup_{s=2}^{m-1} J_{m, s}$ and $f_{i}=\alpha_{m-s}\left(x^{i}-x^{i-a_{m}}\right)$ for $i \in I_{m, s}, s \in[1, m)$ and $f_{i}=\alpha^{\left\langle\left[(2 s-1) a_{m}-2 i\right] / \sqrt{\left.4 a_{m}\right\rangle}\right.} x^{i}$ for $i \in J_{m, s}, s \in[1, m)$. If $j \in M_{m, n}$, then $j \in I_{m, m-n}$; if $i \in\left[a_{m}, c_{m}\right]$ and $i-a_{m} \in M_{m, n}$, then $i \in J_{m, m-n+2}$. Thus $y_{m, j}=\alpha_{n} e_{j}$ for $j \in M_{m, n}$. Clearly, $M_{m, n} \subset\left(v_{m-1},(m-k) a_{m}\right.$ ]. Using (3.2) and (3.4) we obtain for $n>k$

$$
\max _{v_{n-2}<j \leq v_{n-1}}\left|z_{n, j}\right| \leq \max _{m>n} \max _{v_{n-2}<j \leq v_{n-1}}\left|\alpha_{n}\right|\left|e_{j+(m-n) a_{m}}\right|=\max _{m>n} \max _{j \in M_{m, n}}\left|y_{m, j}\right| \leq a_{n+1}^{-1} .
$$

From (3.3) and (3.1) we get for $n>k$

$$
\max _{j \in\left[0, v_{n-1}\right]}\left|y_{n, j}+z_{n, j}\right| \leq\left|P_{a_{n}}\left(Q_{n} e\right)\right|<a_{n}^{-1}
$$

Hence, by (3.5), we have for $n>k$

$$
\max _{j \in\left(v_{n-2}, v_{n-1}\right]}\left|y_{n, j}\right|<a_{n}^{-1}
$$

Let $n>k$. Put $M_{n}=\sum_{j=v_{n-1}+1}^{c_{n}} e_{j} f_{j}-\sum_{j=v_{n-1}+1}^{c_{n}} y_{n, j} x^{j}$. Clearly, $\left(v_{n-1}, c_{n}\right]=$ $\bigcup_{s=1}^{n-1}\left(J_{n, s} \cup I_{n, s}\right)$. If $i \in \bigcup_{s=1}^{n-1} J_{n, s} \cup \bigcup_{s=2}^{n-1} I_{n, s}$, then $P_{v_{n-1}} f_{i}=0$; if $i \in I_{n, 1}$, then $P_{v_{n-1}}\left(f_{i}\right) \in F_{v_{n-2}}$. Thus $P_{v_{n-1}}\left(M_{n}\right) \in F_{v_{n-2}}$; but $M_{n}=\sum_{j=0}^{v_{n-1}} y_{n, j} x^{j}-\sum_{j=0}^{v_{n-1}} e_{j} f_{j} \in$ 
$F_{v_{n-1}}$, so $M_{n} \in F_{v_{n-2}}$. Hence $\sum_{j=v_{n-2}+1}^{v_{n-1}} e_{j} f_{j}-\sum_{j=v_{n-2}+1}^{v_{n-1}} y_{n, j} x^{j}=\sum_{j=0}^{v_{n-2}} t_{n, j} f_{j}$ for some $\left(t_{n, j}\right)_{j=0}^{v_{n-2}} \subset \mathbb{K}$. By (3.7) and Lemma 1 we get

$$
\begin{aligned}
\max _{j \in\left(v_{n-2}, v_{n-1}\right]}\left|e_{j}\right| & \leq\left\|\sum_{j=v_{n-2}+1}^{v_{n-1}} e_{j} f_{j}-\sum_{j=0}^{v_{n-2}} t_{n, j} f_{j}\right\| \leq \max _{j \in\left(v_{n-2}, v_{n-1}\right]}\left|y_{n, j}\right|\left\|x^{j}\right\| \\
& \leq \frac{B_{n-1}}{a_{n}}<\frac{1}{a_{n}^{1 / 2}} .
\end{aligned}
$$

Hence for $m \geq k$ we have $\max _{j \in\left(v_{m-1}, c_{m}\right]}\left|e_{j}\right| \leq \max _{j \in\left(v_{m-1}, v_{m}\right]}\left|e_{j}\right|<a_{m+1}^{-1 / 2}<a_{m}^{-1}$. Using (3.2) we get for $n \geq k$ :

$$
\begin{aligned}
\max _{j \in\left[0, v_{n-1}\right]}\left|z_{n, j}\right| & \leq\left|\alpha_{n}\right| \max _{m>n} \max _{j \in\left[0, v_{n-1}\right]}\left|e_{j+(m-n) a_{m}}\right| \leq\left|\alpha_{n}\right| \max _{m>n} \max _{j \in\left(v_{m-1}, v_{m}\right]}\left|e_{j}\right| \\
& \leq\left|\alpha_{n}\right| \max _{m>n} a_{m+1}^{-1 / 2}=\left|\alpha_{n}\right| a_{n+2}^{-1 / 2} \leq a_{n+1}^{-1} .
\end{aligned}
$$

Let $n \in \mathbb{N}$ with $n>k$. Applying (3.6) we obtain

$$
\left|P_{v_{n-1}}\left(\sum_{j=0}^{c_{n}} e_{j} f_{j}\right)\right|=\left|P_{v_{n-1}}\left(\sum_{j=0}^{c_{n}} y_{n, j} x^{j}\right)\right|=\max _{j \in\left[0, v_{n-1}\right]}\left|y_{n, j}\right|<a_{n}^{-1} .
$$

Moreover we have

$$
\left|P_{v_{n-1}}\left(\sum_{j=v_{n-1}+1}^{c_{n}} e_{j} f_{j}\right)\right| \leq \max _{j \in\left(v_{n-1}, c_{n}\right]}\left|e_{j}\right| \max _{j \in\left(v_{n-1}, c_{n}\right]}\left|P_{v_{n-1}}\left(f_{j}\right)\right| \leq a_{n}^{-1} a_{n-1} .
$$

Thus $\left|\sum_{j=0}^{v_{n-1}} e_{j} f_{j}\right|=\left|P_{v_{n-1}}\left(\sum_{j=0}^{c_{n}} e_{j} f_{j}\right)-P_{v_{n-1}}\left(\sum_{j=v_{n-1}+1}^{c_{n}} e_{j} f_{j}\right)\right|<a_{n}^{-1} a_{n-1}$. For some $\left(s_{n, j}\right)_{j=0}^{v_{n-1}} \subset \mathbb{K}$ we have $\sum_{j=0}^{v_{n-1}} e_{j} f_{j}=\sum_{j=0}^{v_{n-1}} s_{n, j} x^{j}$. Hence

$$
\begin{aligned}
\max _{j \in\left[0, v_{n-1}\right]}\left|e_{j}\right| & =\left\|\sum_{j=0}^{v_{n-1}} s_{n, j} x^{j}\right\| \leq \max _{j \in\left[0, v_{n-1}\right]}\left|s_{n, j}\right|\left\|x^{j}\right\| \leq\left|\sum_{j=0}^{v_{n-1}} s_{n, j} x^{j}\right| B_{n-1} \\
& \leq a_{n}^{-1} a_{n-1} B_{n-1}<a_{n-1}^{-1}
\end{aligned}
$$

for every $n>k$. It follows that $\max _{j \in \mathbb{N}_{0}}\left|e_{j}\right|=0$, so $e=0$, which is a contradiction.

Lemma 6 Let $0<\varepsilon<1$ and $1<M<\varepsilon^{-1}$. Let $n \in \mathbb{N}$ and $m \in[0, n]$. Assume that $y \in F_{n}$ with $|y| \leq M$ and $\left|P_{m}(y)\right| \geq M \varepsilon$. Then there exists $q \in F_{n}$ with $|q| \leq \varepsilon^{-(n+2) !}$ such that $\left|P_{n}(q y)-x^{m}\right|<\varepsilon$.

Proof Clearly, $y=\sum_{i=0}^{n} y_{i} x^{i}$ for some $\left(y_{i}\right)_{i=0}^{n} \subset \mathbb{K}$. By assumptions we have $\max _{0 \leq i \leq n}\left|y_{i}\right| \leq M$ and $\max _{0 \leq i \leq m}\left|y_{i}\right| \geq M \varepsilon$.

If $n=1$ and $m=0$, then $q=y_{0}^{-1} x^{0}-y_{0}^{-2} y_{1} x^{1}$ satisfies our claim. 
If $n=1$ and $m=1$, then we can take $q=y_{1}^{-1} x^{0}$ if $\left|y_{0}\right|<\varepsilon^{2} M$, and $q=y_{0}^{-1} x^{1}$ if $\left|y_{0}\right| \geq \varepsilon^{2} M$.

Suppose that our claim is true for $n=k \geq 1$. We shall prove that it is true for $n=k+1$.

Let $m=0$. Then we put $q_{0}=y_{0}^{-1}$ and $q_{i+1}=-y_{0}^{-1} \sum_{j=1}^{i+1} y_{j} q_{i+1-j}$ for $0 \leq i \leq k$ and $q=\sum_{i=0}^{k+1} q_{i} x^{i}$. It is easy to see that $P_{k+1}(q y)-x^{0}=0$ and

$$
|q|=\max _{0 \leq i \leq k+1}\left|q_{i}\right| \leq \max _{0 \leq i \leq k+1} \varepsilon^{-i}(M \varepsilon)^{-1}=\varepsilon^{-k-2} M^{-1} \leq \varepsilon^{-(k+3) !} .
$$

Let $1 \leq m \leq k+1$. Consider two cases.

Case 1: $\left|y_{0}\right|<\varepsilon^{(k+2) !+1}$. Then $\max _{1 \leq i \leq k+1}\left|y_{i}\right| \leq M$ and $\max _{1 \leq i \leq m}\left|y_{i}\right| \geq M \varepsilon$. By the inductive assumption for $\bar{y}=\sum_{i=1}^{k+1} y_{i} x^{i-1}$, there exists $q=\sum_{i=0}^{k} q_{i} x^{i}$ with $|q| \leq \varepsilon^{-(k+2) !}$ and $\left|P_{k}(q \bar{y})-x^{m-1}\right|<\varepsilon$. Then we have

$$
\left|P_{k+1}(q y)-x^{m}\right|=\left|P_{k+1}\left(q\left(x \bar{y}+y_{0} x^{0}\right)\right)-x x^{m-1}\right|=\left|x\left(P_{k}(q \bar{y})-x^{m-1}\right)+y_{0} q\right|<\varepsilon .
$$

Case 2: $\left|y_{0}\right| \geq \varepsilon^{(k+2) !+1}$. Then we put $q_{i}=0$ for $0 \leq i<m, q_{m}=y_{0}^{-1}$, and $q_{m+j}=-y_{0}^{-1} \sum_{i=1}^{j} y_{i} q_{m+j-i}$ for $1 \leq j \leq k+1-m$. For $q=\sum_{i=0}^{k+1} q_{i} x^{i}$ it is easy to check that $P_{k+1}(q y)-x^{m}=0$ and

$$
|q|=\max _{0 \leq j \leq k+1-m}\left|q_{m+j}\right| \leq \max _{0 \leq j \leq k+1-m} \varepsilon^{-(k+2) !-1}\left(M \varepsilon^{-(k+2) !-1}\right)^{j} \leq \varepsilon^{-(k+3) !} .
$$

Now we are ready to show our main result.

Theorem 7 Assume that $d_{1} \geq|\alpha|^{4}$ and $d_{n+1} \geq|\alpha|^{\left(n d_{n}\right) !}$ for every $n \in \mathbb{N}$. Then the linear continuous operator $T$ on $E$ has no non-trivial closed invariant subspace.

Proof Let $M$ be a closed subspace of $E$ with $M \neq\{0\}$ such that $T(M) \subset M$. Then $g(T)(M) \subset M$ for every $g \in F$. Let $e \in M$ with $0<\|e\| \leq 1$. We shall prove that for every $\delta>0$ there exists $f \in F$ such that $\left\|f(T) e-x^{0}\right\|<\delta$. Let $\delta>0$. Let $k>2$ with $a_{k-1}>|\alpha| \delta^{-1}$. By Lemma 5 we have $\left|P_{(m-k) a_{m}}\left(Q_{m} e\right)\right| \geq a_{m}^{-1}$ for some $m>k$. For $R_{m}=\left(a_{m} A_{m}\right)^{\left[(m-2) a_{m}+2\right] !}$ we have $|\alpha| a_{m} A_{m} R_{m}<b_{m}$, since $a_{m} A_{m}<|\alpha|^{a_{m}}$ and $|\alpha|^{\left(m a_{m}\right) !}<b_{m}$. Put $y=Q_{m} e$. Then $|y| \leq \sqrt{A_{m}}\left\|Q_{m}\right\|\|e\| \leq A_{m}$. By Lemma 6 and its proof there exists $q \in F_{(m-2) a_{m}}$ with $|q| \leq R_{m}$ such that

$$
\left|P_{(m-2) a_{m}}(q y)-x^{(m-k) a_{m}}\right|<\left(a_{m} A_{m}\right)^{-1} .
$$

(S1) Put $f=\beta_{m}^{-1} x^{a_{m}+b_{m}} q$ and $S=K_{m, 1}$. Then $f=\sum_{s \in S} t_{s} x^{s}$ for some $\left(t_{s}\right)_{s \in S} \subset \mathbb{K}$. Let $z=f y$. Using Lemma 4 we get

$$
\begin{aligned}
\|f(T) e-z\| & =\left\|\sum_{s \in S} t_{s}\left(T^{s}-T^{s} Q_{m}\right) e\right\| \leq \max _{s \in S}\left|t_{s}\|\alpha|=| f\| \alpha\right| \\
& =\left|q\|\alpha\| \beta_{m}\right|^{-1}<R_{m}\left|\alpha \| \beta_{m}\right|^{-1}<a_{m}^{-1} .
\end{aligned}
$$


(S2) Let $a=(m-1) a_{m}+b_{m}$ and $b=2(m-1) a_{m}+b_{m}$. Clearly $z \in F_{b}$; so $z=\sum_{j=0}^{b} s_{j} x^{j}$ for some $\left(s_{j}\right)_{j=0}^{b} \subset \mathbb{K}$. Then $\left\|z-P_{a} z\right\|=\left\|\sum_{j=a+1}^{b} s_{j} x^{j}\right\| \leq$ $\max _{a<j \leq b}\left|s_{j}\right| \max _{a<j \leq b}\left\|x^{j}\right\| \leq|z|$, since

$$
\left\|x^{j}\right\|=|\alpha|^{-\left\langle\left[3 b_{m}-2 j\right] / \sqrt{4 b_{m}}\right\rangle} \leq|\alpha|^{-\left\langle\left[b_{m}-4(m-1) a_{m}\right] / \sqrt{4 b_{m}}\right\rangle} \leq 1
$$

for $j \in(a, b] \subset L_{m, 2}$. We have $|z|=\left|f\left\|y|=| \beta_{m}^{-1}\right\| q \| y\right| \leq\left|\beta_{m}^{-1}\right| R_{m} A_{m}<a_{m}^{-1}$. Thus $\left\|z-P_{a} z\right\|<a_{m}^{-1}$.

(S3) Let $t=x^{a_{m}} q y$ and $c=(m-1) a_{m}$. Clearly $t \in F_{2 c}$; so $t=\sum_{j=a_{m}}^{2 c} \gamma_{j} x^{j}$ for some $\left(\gamma_{j}\right)_{j=a_{m}}^{2 c} \subset \mathbb{K}$. For $j \in\left[a_{m}, c\right]$ we have $j+b_{m} \in K_{m, 1}$. Thus $\left\|\beta_{m}^{-1} x^{j+b_{m}}-x^{j}\right\|=$ $\left|\beta_{m}^{-1} \|\right| f_{j+b_{m}}|=| \beta_{m}^{-1} \mid$. Hence we get

$$
\begin{aligned}
\left\|P_{a} z-P_{c} t\right\| & =\left\|P_{a}\left(\beta_{m}^{-1} x^{b_{m}} t\right)-P_{c} t\right\|=\left\|P_{a}\left(\sum_{j=a_{m}}^{2 c} \gamma_{j} \beta_{m}^{-1} x^{j+b_{m}}\right)-P_{c}\left(\sum_{j=a_{m}}^{2 c} \gamma_{j} x^{j}\right)\right\| \\
& =\left\|\sum_{j=a_{m}}^{c} \gamma_{j} \beta_{m}^{-1} x^{j+b_{m}}-\sum_{j=a_{m}}^{c} \gamma_{j} x^{j}\right\| \leq \max _{a_{m} \leq j \leq c}\left|\gamma_{j}\right|\left\|\beta_{m}^{-1} x^{j+b_{m}}-x^{j}\right\| \\
& \leq|t|\left|\beta_{m}^{-1}\right| .
\end{aligned}
$$

Thus $\left\|P_{a} z-P_{c} t\right\| \leq\left|\beta_{m}^{-1}\right| R_{m} A_{m}<a_{m}^{-1}$, since $|t|=|q||y| \leq R_{m} A_{m}$.

(S4) Using (3.8) we get

$$
\begin{aligned}
\left|P_{c} t-x^{(m-k+1) a_{m}}\right| & =\left|x^{a_{m}}\left(P_{c-a_{m}}(q y)-x^{(m-k) a_{m}}\right)\right| \\
& =\left|P_{(m-2) a_{m}}(q y)-x^{(m-k) a_{m}}\right|<\left(a_{m} A_{m}\right)^{-1} .
\end{aligned}
$$

Hence $\left\|P_{c} t-x^{(m-k+1) a_{m}}\right\| \leq a_{m}^{-1}$.

(S5) By Lemma 1 we have $\left\|x^{(m-k+1) a_{m}}-x^{0}\right\|<|\alpha| a_{k-1}^{-1}$. Since

$f(T) e-x^{0}=(f(T) e-z)+\left(z-P_{a} z\right)+\left(P_{a} z-P_{c} t\right)+\left(P_{c} t-x^{(m-k+1) a_{m}}\right)+\left(x^{(m-k+1) a_{m}}-x^{0}\right)$,

we obtain $\left\|f(T) e-x^{0}\right\| \leq|\alpha| a_{k-1}^{-1}<\delta$.

We have shown that for every $\delta>0$ there exists $f \in F$ such that $\left\|f(T) e-x^{0}\right\|<\delta$. It follows that $x^{0} \in M$. Hence $x^{n}=T^{n} x^{0} \in M$ for all $n \in \mathbb{N}$. Thus $F \subset M$, so $M=E$.

\section{References}

[1] B. Beauzamy, Introduction to Operator Theory and Invariant Subspaces. North-Holland Mathematical Library 42, North-Holland, Amsterdam, (1988.

[2] P. Enflo, On the invariant subspace problem for Banach spaces. Acta Math. 158(1987), no. 3-4, 212-313.

[3] J. Lindenstrauss and L. Tzafriri, On complemented subspaces problem. Israel J. Math. 9(1971), 263-269.

[4] J. B. Prolla, Topics in Functional Analysis over Valued Division Rings. North-Holland Mathematics Studies 77, North-Holland, Amsterdam, 1982. 
[5] C. J. Read, A solution to the invariant subspace problem. Bull. London Math. Soc. 16(1984), no. 4, 337-401.

[6] A solution to the invariant subspace problem on the space $l_{1}$. Bull. London Math. Soc. 17(1985), no. 4, 305-317.

[7] A short proof concerning the invariant subspace problem. J. London Math. Soc. 34(1986), no. 2, 335-348.

[8] A. C. M. van Rooij, Non-Archimedean Functional Analysis. Monographs and Textbooks in Pure and Applied Math. 51, Marcel Dekker, New York, 1978.

[9] A. C. M. van Rooij and W. H. Schikhof, Open problems. In: p-Adic Functional Analysis. Lecture Notes in Pure and Appl. Math. 137, Dekker, New York, 1992, pp. 209-219.

[10] P. Schneider, Nonarchimedean Functional Analysis. Springer-Verlag, Berlin, 2002.

Faculty of Mathematics and Computer Science, A. Mickiewicz University, 61-614 Poznań, Poland e-mail: sliwa@amu.edu.pl 